PHYSICAL REVIEW FLUIDS 2, 039901(E) (2017)

\title{
Publisher's Note: Lubricated wrinkles: Imposed constraints affect the dynamics of wrinkle coarsening [Phys. Rev. Fluids 2, 014202 (2017)]
}

\author{
Ousmane Kodio, Ian M. Griffiths, and Dominic Vella
}

(Received 23 March 2017; published 29 March 2017)

DOI: 10.1103/PhysRevFluids.2.039901

This paper was published online on 23 January 2017 with an error in the title of Ref. 6 . Reference 6 should read as "Y. Matia and A. D. Gat, Dynamics of elastic beams with embedded fluid-filled parallel-channel networks, Soft Robotics 2, 42 (2015)." The reference has been corrected as of 23 March 2017. 